\title{
Journal of Real-Time Image Processing: second issue of volume 14
}

\author{
Nasser Kehtarnavaz ${ }^{1} \cdot$ Matthias F. Carlsohn ${ }^{2}$ \\ Published online: 5 March 2018 \\ (C) Springer-Verlag GmbH Germany, part of Springer Nature 2018
}

As was mentioned in a previous editorial, this year in 2018 two volumes are scheduled to be published in print: volume 14 and volume 15. Each volume will consist of 4 issues with each issue comprising 200 pages or so. The first issue of volume 14 was a special issue and has already appeared. This is the second issue of volume 14 which is a regular issue consisting of 15 papers in the order they appeared online on the journal website. The purpose of offering two volumes is to reduce the print backlog of the papers that currently appear online. The next or third issue of volume 14 is planned to be a combination of two special issues, one special issue on "Heterogeneous Image Processing" together with another special issue on "Advances on Smart Camera Architectures for Real-Time Image Processing". The fourth issue of volume 14 will be a regular issue which will complete volume 14 half way through the year. Then volume 15 will run in the second half of the year.

As noted in a previous editorial, to provide an incentive to our reviewers, for those reviewers who carry out $5+$ high quality reviews, a certificate of recognition will be issued by Springer. In addition, such reviewers will be offered a complimentary Springer book. Those reviewers who complete $10+$ high quality reviewers will be considered to join the JRTIP editorial board as an Associate Editor. For more information on this so called Reviewer Reward Program, the readers are referred to this website: http://www.springer.com/computer/image+ processing/jour nal/11554/PSE?detailsPage=press

As has been practiced for many years, the JRTIP editorial board will be meeting on April 16 in Orlando, FL, during the SPIE Conference on Real-Time Image and Video Processing which is now a part of the SPIE

Nasser Kehtarnavaz

kehtar@utdallas.edu

Matthias F. Carlsohn

Matthias.carlsohn@t-online.de

University of Texas at Dallas, Richardson, TX, USA

2 Engineering and Consultancy for Computer Vision and Image Communication, Bremen, Germany
Symposium on Defense and Commercial Sensing. The real-time image and video processing papers to be presented at the conference can be seen at this website: http:// spie.org/SIC/conferencedetails/real-time-image-video-pro cessing? $\mathrm{SSO}=1$. The outcome of this editorial board meeting will be reported in a later editorial.

This regular issue consists of 15 papers which address different aspects of real-time image and video processing.

The first paper by Silveira et al. is entitled "Reference frame context-adaptive variable-length coder: a real-time hardware-friendly approach for lossless external memory bandwidth reduction in current video-coding systems." This paper presents the Reference Frame Context Adaptive Variable-Length Coder (named RFCAVLC) as a lowcomplexity lossless solution to compress data before storing in external memory. The solution presented uses Huffman codes and eight static code tables. The RFCAVLC design presented is shown to provide real-time encoding for WQSXGA $(3200 \times 2048$ pixels $)$ images at $33 \mathrm{fps}$.

The second paper by Acharya et al. is entitled "A realtime implementation of SIFT using GPU." This paper addresses the high computational complexity associated with the widely used Scale-Invariant Feature Transform (SIFT) algorithm. A parallel implementation of SIFT on a GPU is presented where a speed of around $55 \mathrm{fps}$ for images of size $640 \times 480$ is achieved. A major contribution of this work is the introduction of a combined kernel optimization leading to more than $12 \%$ improvement in the execution speed.

The third paper by Gadeski et al. is entitled "GPU deformable part model for object recognition." This paper considers the problem of rapidly detecting objects in images or video. A GPU implementation is discussed which generates comparable classification performance to existing techniques while achieving more than 10 times speedup compared to a baseline implemented in $\mathrm{C}++$ on a single core and 5 times compared to a multi-core OpenMP implementation.

The fourth paper by Jiang is entitled "Real-time multiresolution edge detection with pattern analysis on graphics 
processing unit." This paper discusses some general rules for edge representation based upon the classification of edge types into the four categories of ramp, impulse, step, and sigmoid. The paper presents a multi-resolution edge detection algorithm with edge pattern analysis and covers a parallel implementation on a GPU. It is shown that the GPU accelerated sigmoid implementation provides a scalable speedup as the resolution of images is increased.

The fifth paper by Amamra et al. is entitled "GPU-based real-time RGBD data filtering." This paper presents an adaptation of the Kalman filtering scheme to improve the precision of Kinect depth cameras as real-time RGBD sensors. The proposed Kalman filter adaptation to any Kinect-like camera is demonstrated via experiments and a GPU implementation of the filter with different optimization levels is also presented.

The sixth paper by Berg et al. is entitled "Highly efficient image registration for embedded systems using a distributed multicore DSP architecture." This paper describes an efficient image registration approach for embedded systems. An implementation on an embedded distributed multicore digital signal processor (DSP) architecture is covered based on four TI C6678 DSPs with eight cores each. It is shown that two images of size $4096 \times 4096$ pixels are registered within $93 \mathrm{~ms}$ with 3 times less power consumption.

The seventh paper by Soua et al. is entitled "GPU parallel implementation of the new hybrid binarization based on Kmeans method (HBK)." This paper discusses a parallel implementation of the hybrid binarization based Kmeans (named HBK) method on the NVIDIA GTX 660 GPU. The implementation combines fine-grained and coarse-grained parallelism strategies and avoids CPU-GPU communication overhead. It is shown that the binarization of one document is achieved in $425 \mathrm{~ms}$, meeting the targeted real-time optical character recognition (OCR) system.

The eighth paper by Craciun et al. is entitled "A realtime, power-efficient architecture for mean-shift image segmentation." This paper presents how to address the computational complexity of the mean-shift algorithm for performing unsupervised clustering in image segmentation applications. A hardware architecture is proposed that exploits the computing power of FPGAs by clustering pixels in parallel. This architecture is compared with the existing CPU and GPU implementations and its advantages over these implementations in terms of both execution time and power consumption are demonstrated.

The ninth paper by Jiang et al. is entitled "A dynamically reconfigurable architecture system for time-varying image constraints (DRASTIC) for motion JPEG." This paper discusses a dynamically reconfigurable system for time-varying image constraints (named DRASTIC) for video communication applications. Based on a hardwaresoftware co-design approach, a family of scalable 2D DCT hardware modules is defined which gets optimized in software. For full 2D DCT computation, the results show that the proposed implementation is as good as or better than previous implementations. In addition, a scalable, real-time controller is considered for selecting an appropriate configuration.

The tenth paper by Hossam et al. is entitled "Accelerated hyperspectral image recursive hierarchical segmentation using GPUs, multicore CPUs, and hybrid CPU/GPU cluster." This paper provides accelerated parallel solutions for the recursive hierarchical segmentation (named RHSEG) algorithm on a GPU, a hybrid multicore CPU with GPU, and a hybrid multicore CPU/GPU cluster. Compared to the CPU sequential implementation, it is shown that these parallel solutions provide speedups of 21 times for the parallel single GPU implementation and 240 times for the hybrid multinode implementation.

The eleventh paper by Bahaoui et al. is entitled "Blocking artifact removal using partial overlapping based on exact Legendre moments computation." This paper discusses the design of a partial overlapping block using exact Legendre moment computation (named POBRELM) for gray-level image reconstruction. The proposed reconstruction method takes advantage of only partial information of the neighbors of each block. The simulation results indicate that not only an improvement is made in terms of the reconstruction error, but also a considerable amount of reduction is achieved in the computation time.

The twelfth paper by Cano et al. is entitled "Parallelization strategies for markerless human motion capture." This paper presents an approach to parallelize the markerless motion capture (named MMOCAP) algorithm in CPUs and GPUs. This approach is experimentally compared on sequences of the HumanEva-I dataset. It is shown that the proposed approach achieves 8 times speedups in multi-core CPUs, 30 times in a single GPU, and up to 110 times when using 4 GPUs.

The thirteenth paper by Raj et al. is entitled "Video-rate calculation of depth from defocus on a FPGA." This paper addresses the FPGA implementation of a depth from defocus algorithm. The multiplier and SRAM features of the FPGA are utilized to enable an efficient implementation by exploiting the symmetry of the filter used in the algorithm. It is shown that this implementation allows range images to be processed within $14 \mathrm{~ms}$.

The fourteenth paper by Yao et al. is entitled "Real-time stereo to multi-view conversion system based on adaptive meshing." This paper focuses on a real-time GPU implementation of the stereo to multi-view conversion pipeline. To reduce the computational complexity, an adaptive meshing, a simple block matching, and fine-grained 
optimization techniques are deployed. The results obtained indicate that real-time and semi-real-time performances are achieved when rendering 8 views with the image resolutions of $1280 \times 720$ and $1920 \times 1080$ on Tesla K20 GPU.

The fifteenth or the final paper by Jung et al. is entitled "A fast deconvolution-based approach for single-image super-resolution with GPU acceleration." This paper presents a fast deconvolution-based image super-resolution approach for GPU implementation. An efficient deconvolution method and a directly parallelizable technique are proposed. The experimental results demonstrate that the proposed approach achieves better performance as compared to the existing approaches in terms of both image quality and runtime. 